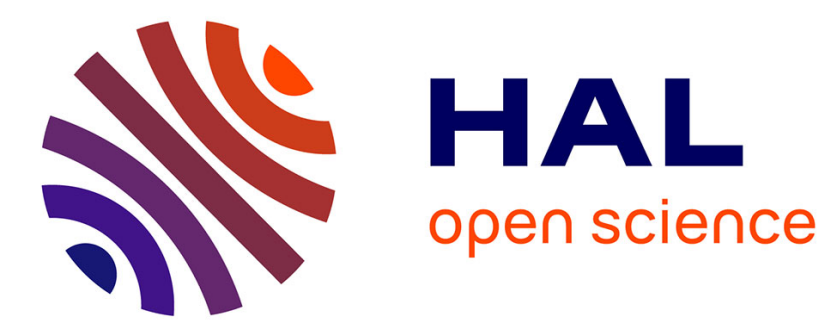

\title{
Biomedical image segmentation using geometric deformable models and metaheuristics
}

Pablo Mesejo, Andrea Valsecchi, Linda Marrakchi-Kacem, Stefano Cagnoni, Sergio Damas

\section{- To cite this version:}

Pablo Mesejo, Andrea Valsecchi, Linda Marrakchi-Kacem, Stefano Cagnoni, Sergio Damas. Biomedical image segmentation using geometric deformable models and metaheuristics. Computerized Medical Imaging and Graphics, 2015, 43, pp.167-178. 10.1016/j.compmedimag.2013.12.005 hal-01221316v3

\section{HAL Id: hal-01221316 https://inria.hal.science/hal-01221316v3}

Submitted on 8 Mar 2016

HAL is a multi-disciplinary open access archive for the deposit and dissemination of scientific research documents, whether they are published or not. The documents may come from teaching and research institutions in France or abroad, or from public or private research centers.
L'archive ouverte pluridisciplinaire HAL, est destinée au dépôt et à la diffusion de documents scientifiques de niveau recherche, publiés ou non, émanant des établissements d'enseignement et de recherche français ou étrangers, des laboratoires publics ou privés. 


\title{
Biomedical Image Segmentation using Geometric Deformable Models and Metaheuristics
}

\author{
Pablo Mesejo $^{\mathrm{a}}$, Andrea Valsecchi ${ }^{\mathrm{b}}$, Linda Marrakchi-Kacem $^{\mathrm{c}, \mathrm{d}}$, Stefano Cagnoni ${ }^{\mathrm{a}}$, Sergio Damas ${ }^{\mathrm{b}}$ \\ ${ }^{a}$ Intelligent Bio-Inspired Systems laboratory (IBISlab), \\ Department of Information Engineering, \\ University of Parma, Viale G.P. Usberti 181a, 43124, Parma, Italy \\ ${ }^{b}$ European Center for Soft Computing, \\ C/ Gonzalo Gutiérrez Quirós, s/n- $3^{a}$ planta, 33600 Mieres, Spain \\ ${ }^{c}$ Neurospin, CEA, Gif-Sur-Yvette, France \\ ${ }^{d}$ CRICM, UPMC Université Paris 6, France
}

\begin{abstract}
This paper describes a hybrid level set approach for medical image segmentation. This new geometric deformable model combines region- and edge-based information with the prior shape knowledge introduced using deformable registration. Our proposal consists of two phases: training and test. The former implies the learning of the level set parameters by means of a Genetic Algorithm, while the latter is the proper segmentation, where another metaheuristic, in this case Scatter Search, derives the shape prior. In an experimental comparison, this approach has shown a better performance than a number of state-of-the-art methods when segmenting anatomical structures from different biomedical image modalities.
\end{abstract}

Keywords: Image Segmentation, Deformable Models, Deformable Registration, Genetic Algorithms, Scatter Search

\section{Introduction}

Image segmentation is commonly defined as the partitioning of an image into non-overlapping regions that are homogeneous with respect to some visual feature, such as color or texture [1]. In many medical imaging applications, segmentation algorithms play a crucial role by automatically identifying anatomical structures and other regions of interest. Such algorithms are nowadays in the core of multiple tasks, like quantification and measurement of tissue volumes, localization of pathologies or computerintegrated surgery. It is important to highlight that manual segmentation is not only tedious and time consuming but, sometimes, also inaccurate, hence the importance of developing automatic and accurate segmentation methods.

In particular, medical imaging segmentation is usually challenging due to poor image contrast, noise, diffuse organ/tissue boundaries, and artifacts. These problems can cause considerable difficulties when applying traditional segmentation techniques, such as edge detection or thresholding. Consequently, an intelligent way of proceeding is to incorporate as much prior knowledge as possible about the particular object and image modality to segment. To

\footnotetext{
Email addresses: pmesejo@ce.unipr.it (Pablo Mesejo), andrea.valsecchi@softcomputing.es (Andrea Valsecchi), linda.marrakchi@gmail.com (Linda Marrakchi-Kacem), cagnoni@ce.unipr.it (Stefano Cagnoni), sergio.damas@softcomputing.es (Sergio Damas)
}

address these difficulties, deformable models have been extensively studied and widely used in medical image segmentation with interesting results $[2,3]$.

A single source of prior knowledge is usually not enough to satisfactorily tackle medical image segmentation problems. Therefore, the development of hybrid approaches combining different sources of information has been a major focus in the field of image segmentation $[4,5,6]$. In this work, the search/learning abilities of metaheuristics and the capability of geometric deformable models to handle topological changes are combined. Three sources of information (a region term, a shape prior, and an edge term) are used to accurately segment the organs of interest in different medical image modalities: microscopy, Xray computed tomography $(\mathrm{CT})$, and magnetic resonance imaging (MRI). In our proposal, metaheuristics [7] have capital importance in two stages. First, during the training process of the new model, the tuning of the parameters is carried out by a Genetic Algorithm [8]. Second, in the proper segmentation stage, the shape prior is obtained by a deformable registration process guided by Scatter Search [9].

Every image modality has its own peculiarities, thus the training phase allows our model to learn the most suitable parameters for a specific modality/anatomical district using few images as paradigmatic examples. In turn, the segmentation phase uses only one manually segmented reference image to generate the prior shape knowledge that will guide, together with the region- and edge-based terms, 
the evolution of the moving contour.

To assess the quality of the new approach, we developed an experimental comparison including seven state-ofthe-art segmentation methods. The study was carried out on four different datasets, for a total of 22 microscopy, 11 $\mathrm{MR}$, and $5 \mathrm{CT}$ images.

This paper is structured as follows: in section 2 we provide the theoretical foundations necessary to understand our work. In section 3, a general overview of the method is presented, providing details about the different terms used in our deformable model. Finally, section 4 presents the results and the statistical analysis of the experimental comparison, followed, in section 5 , by some final remarks and a discussion about possible future developments.

\section{Theoretical Background}

In this section, an overview of the main techniques applied in our approach (geometric deformable models, image registration and metaheuristics) and previous related work are presented.

\subsection{Deformable Models}

The term "deformable models" (DMs) was first used in the late eighties [10] with reference to curves or surfaces, defined within the image domain, that are deformed under the influence of "internal" forces, related with the curve features, and "external" forces, related with the features of the image regions surrounding the curve. Internal forces enforce regularity constraints and keep the model smooth during deformation, while external forces are defined to attract the model toward features of the object of interest.

DMs are segmentation techniques that use prior information about the shape of the object to be located or segmented. They start with some initial boundary shape represented in the form of a curve, and iteratively modify it by applying various shrink/expansion operations according to some energy function. DMs can either be regionbased or edge-based approaches, depending on the feature they rely on to segment the object of interest. Regionbased methods usually proceed by partitioning the image into connected regions by grouping neighboring pixels with similar features. Edge-based methods, instead, are focused on contour detection, relying on discontinuities in image values between distinct regions.

There are basically two types of DM depending on the kind of shape representation used: parametric/explicit and geometric/implicit.

- Parametric Deformable Models. This type of DM represents curves and surfaces explicitly in their parametric forms during deformation, allowing direct interaction with the model and leading to a compact representation for fast real-time implementation. As examples of parametric DMs we could cite "snakes" or Active Contour Models (ACMs) [11], Active Shape
Models (ASMs) [12], Active Appearance Models [13, 14], and Topological Active Nets (TANs) [15].

- Geometric Deformable Models. Geometric DMs are based on curve evolution theory $[16,17,18]$ and the Level Set method [19, 20]: curves and surfaces are adapted using only geometric measures, resulting in deformations that are independent of the parameterization but, as in parametric DMs, also rely on image data to delineate object boundaries. Since the adaptation is independent of parameterization, the evolving curves and surfaces can be represented implicitly as a level set of a higher-dimensional function and topological changes can be handled automatically.

Among geometric models, the Level Set (LS) method [19] relies on an evolving closed surface defined by a moving interface, the front, which expands into the image. The interface $\Gamma(t)$ can be characterized as a Lipschitz continuous function:

$$
\begin{cases}\phi(t, \mathbf{x})>0 & \text { for } \mathbf{x} \text { inside } \Gamma(t) \\ \phi(t, \mathbf{x})<0 & \text { for } \mathbf{x} \text { outside } \Gamma(t) \\ \phi(t, \mathbf{x})=0 & \text { for } \mathbf{x} \text { on } \Gamma(t)\end{cases}
$$

The front or evolving boundary, denoted by $\Gamma$, is represented by the zero level $\Gamma(t)=\{\mathbf{x} \mid \phi(t, \mathbf{x})=0\}$ of a LS function $\phi(t, \mathbf{x})$. The dynamics of $\phi$ can be described by the following general form:

$$
\frac{\partial \phi}{\partial t}+F|\nabla \phi|=0
$$

known as the LS equation, where $F$ is called the speed function and $\nabla$ is the spatial gradient operator. $F$ can depend on position, time, the geometry of the interface (e.g., its normal or its mean curvature), or the different image features.

In any case, the definition of the LS function $\phi$ is essential. One common choice is the signed distance function $d(\mathbf{x})$, which gives the distance of a point to the surface and the sign: generally $d>0$ if the point $\mathbf{x}$ is outside and $d<0$ if it is inside the surface (assuming it is a closed surface). This definition is especially interesting to avoid numerical instabilities and inaccuracies during computations. But even with this definition, $\phi$ will not remain a signed distance function all the time and a reinitialization procedure to keep the LS intact will be needed [21].

\subsection{Metaheuristics}

The classic gradient search techniques perform efficiently when the problem under consideration satisfies tight constraints. But when the search space is discontinuous, noisy, high-dimensional and multimodal, then metaheuristics [7] have been found to consistently outperform traditional methods. Among the stochastic approaches to continuous optimization, Evolutionary Algorithms (EAs) and Swarm Intelligence (SI) algorithms, as well as other metaheuristics [22], offer a number of attractive features: no requirement 
for a differentiable or continuous objective function, robust and reliable performance, global search capability, virtually no need of specific information about the problem to solve, easy implementation, and implicit parallelism.

\subsubsection{Genetic Algorithms}

Genetic Algorithms (GAs) [8] are stochastic, parallel search algorithms based on the principles of natural selection. GAs were designed to efficiently search large, non-linear, poorly-understood search spaces where expert knowledge is scarce or difficult to encode and where traditional optimization techniques fail. They are flexible, robust, and try to exhibit the adaptiveness of biological systems.

These algorithms encode a potential solution to a specific problem into a chromosome-like data structure and apply recombination operators to preserve critical information. The main features of a GA are the encoding of individuals as strings of symbols, the individuals selection policy, and the use of both the mutation and recombination operators. The basic outline of a GA is shown in Algorithm 1.

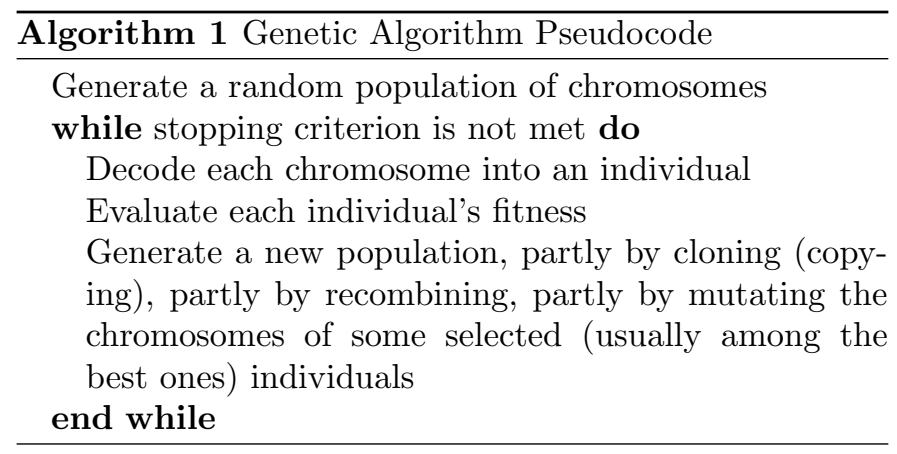

\subsubsection{Scatter Search}

Scatter Search (SS) [9] is based on a systematic combination between solutions (instead of randomized, as is usual in EAs) taken from a subset of the population, named the "reference set", that is usually significantly smaller than a typical EA population. SS is composed of five structural "blocks" or methods:

1. Diversification Generation: a population of solutions $P$ is built with a certain degree of quality and diversity. The reference set $R$ is then drawn from $P$, and is composed of the $\left|R_{1}\right|$ solutions with best fitness, and the $\left|R_{2}\right|$ solutions from $P$ (hence, $|R|=\left|R_{1}\right|+\left|R_{2}\right|$ ) that are farthest, based on a particular metric (usually the Euclidean distance), from the reference set; the evolution process acts only on $R$;

2. Solution Combination: in most problems a specific solution combination method is needed, which can be applied to all solutions or only to selected ones (e.g., the best solutions, and/or randomly selected ones). In many cases an existing crossover operator, borrowed from other EAs, can be employed;
3. Subset Generation: the procedure deterministically generates subsets of $R$, to which the combination method is applied.

4. Improvement: to obtain high-quality solutions, an improvement method (typically a local search method) is applied to the original solutions and/or to combined solutions;

5. Reference Set Update: once a new solution is obtained (applying the combination method) it replaces the worst solution in $\mathrm{R}$ only if it improves the quality of the reference set in terms of fitness and/or diversity;

\subsection{Image Registration}

Image registration (IR) refers to the process of geometrically aligning multiple images having a shared content [23]. The alignment is represented by a spatial transformation that overlaps the common part of the images. One image, the scene, is transformed to match the geometry of the other image, called the model.

Three main components characterize an IR method: the transformation model, the similarity metric and the optimization process. The transformation model determines what kind of transformations can be used to align the images. Transformation models vary greatly in complexity, ranging from simple combinations of translation and rotation up to elastic transformations that can represent local deformations and warpings.

The similarity metric is the component that measures the quality of an alignment. In medical applications, the most common approach, called intensity-based, compares the joint distribution of intensity values between the scene and the model once a transformation has been applied. The degree of matching can be computed from the intensity distributions using measures such as the mean square error, the correlation coefficient or the mutual information [24].

The optimization procedure is the component responsible for finding an appropriate transformation to carry out the registration. A transformation is specified by a series of parameters (e.g. a translation vector and a rotation angle), which turns the registration into a continuous optimization problem. Classic numerical optimization algorithms such as gradient descent, Newton's method, Powell's method and discrete optimization [25, 26] are among the most common choices for the optimization component, as well as approaches based on EAs and other metaheuristics [27].

In this study, image registration is used as a preliminary step in a segmentation process. We assume to have an atlas available (i.e., a typical or average image of the anatomical region to be segmented), in which the target region has been already labeled. The atlas-based segmentation process [28] begins by registering the atlas to the input image. Then, the region of the target image that overlaps the labeled region in the atlas is the result of the 
segmentation process. See Figure 1 for an example of this procedure.
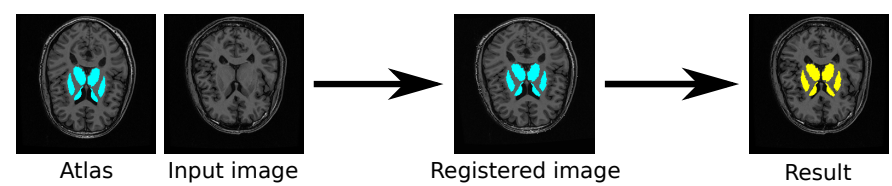

Figure 1: An example of atlas-based segmentation. The figure shows a slice of a 3D brain MRI and the corresponding deep brain structure segmentation obtained once the atlas is registered to the input image.

The quality of atlas-based segmentation depends closely on the accuracy of the registration step, although the anatomical variability in the target region can limit the effectiveness of the method.

\subsection{Image Segmentation using Deformable Models and Meta- heuristics}

It is fundamental to understand that the combination of internal and external forces in a DM determines a target function to minimize, whose minimum is theoretically located at the boundary of the object to segment. This target function can be very complex (noisy, highly-multimodal) and the classic algorithms fail at minimizing it [29]. Hence, the global search capabilities of metaheuristics can be very beneficial to optimize this function. Furthermore, the automatic learning of DM parameters is also possible with these intelligent techniques [30]. In fact, this automatic parameter configuration is even desirable since it is known that manual parameter tuning is time consuming and may introduce a bias in comparing an algorithm with a reference, due to better knowledge of the algorithm under consideration with respect to the reference, or to possible different time spent tuning each of them.

In the literature several examples can be found which hybridize parametric DMs and metaheuristics. In [31] and [32], snakes are combined with an optimization procedure based on GAs. In [33] a GA evolves a population of medialbased shapes extracted from a training set, using prior shape knowledge to produce feasible deformations while also controlling the scale and localization of these deformations. In [34] an ACM is applied to the automatic segmentation of PET images of liver; a GA is used to find optimal parameters values for the edge detection step. Also, in [29], different metaheuristics are compared (Particle Swarm Optimization, SS, GA, Simulated Annealing, Differential Evolution) to optimize an ASM and localize the hippocampus in microscopy images. With respect to standard and extended TANs, in [35], [36] and [37], the minimization of TANs energy to segment CT images is carried out by means of GA and memetic algorithms, Differential Evolution and SS, respectively.

In relation to geometric DMs, much fewer proposals of hybridization have been presented. In [38] a GA is used to perform LS curve evolution using texture and shape information to automatically segment the prostate in CT and MRI pelvic images. In [30], a GA is used to find an optimal set of parameters that characterize the LS method in CT and MRI segmentation. In [39], the initial segmentation based on the LS method is refined using swarms of intelligent agents. Finally, in [40], a comparative study on the segmentation of histological images is carried out where different geometric approaches are initialized using metaheuristics and parametric DMs.

\section{HybridLS method}

In this section, we present a novel segmentation approach based on the LS method, called HybridLS, that combines edge, region and prior shape knowledge of the target object to guide the LS evolution. Moreover, we take advantage of the beneficial characteristics of metaheuristics to automatically learn the inherent parameters of a specific type of object using training data (a set of already segmented images).
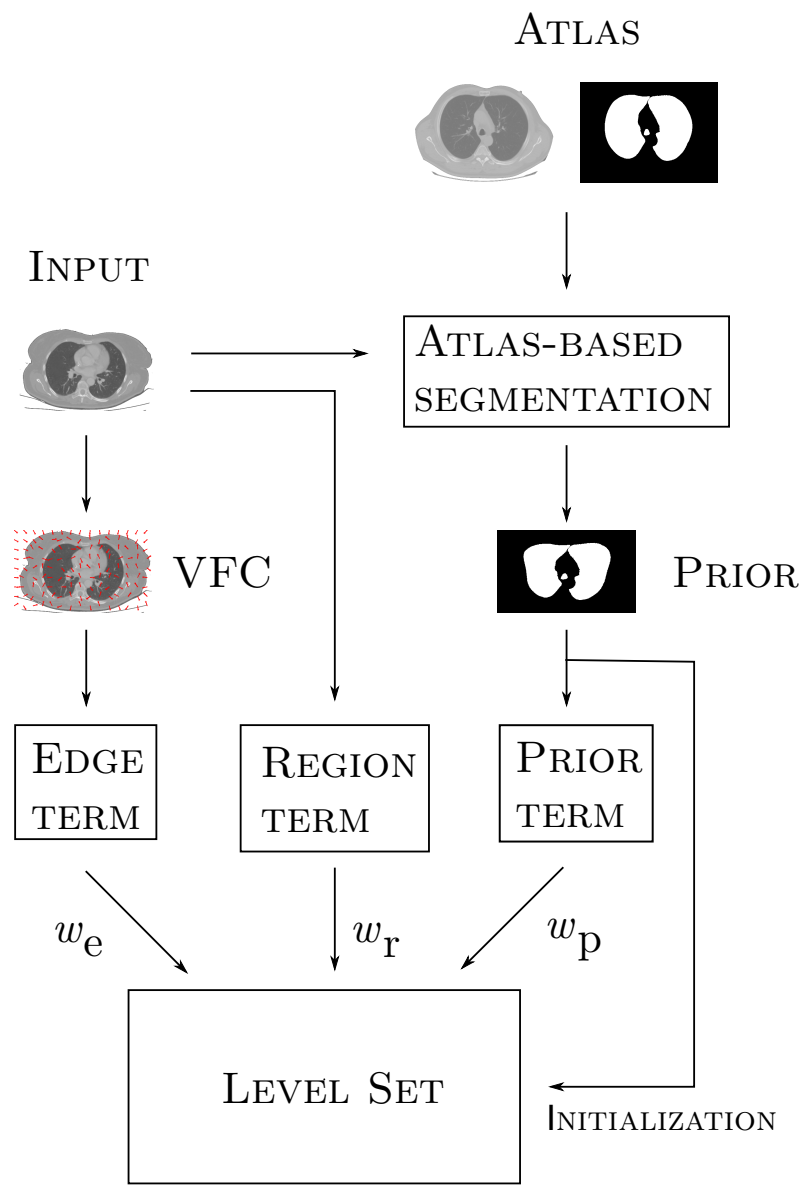

Figure 3: The schematic view of the interaction among the components of HybridLS.

In its first stage, using an atlas of the target object, HybridLS performs atlas-based segmentation of the image under consideration, as in section 2.3. This requires 


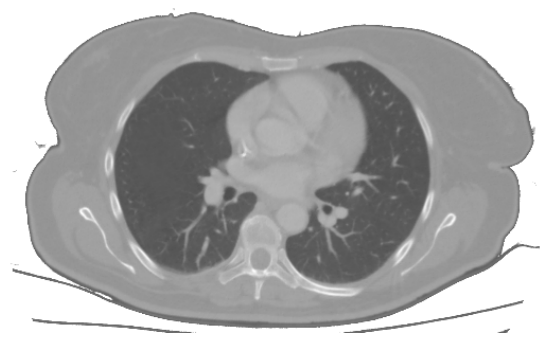

(a) input image

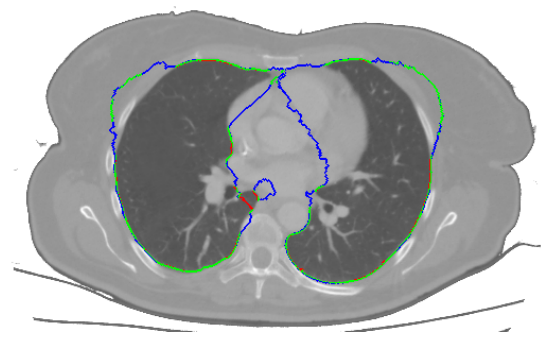

(d) region term

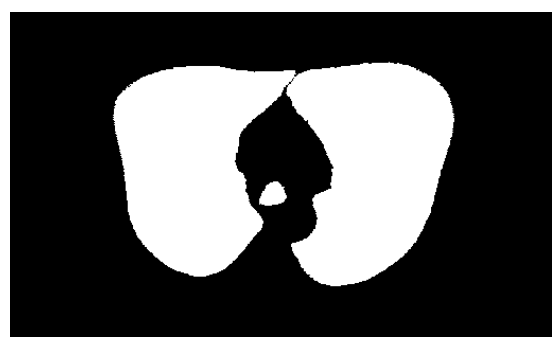

(b) atlas-based prior

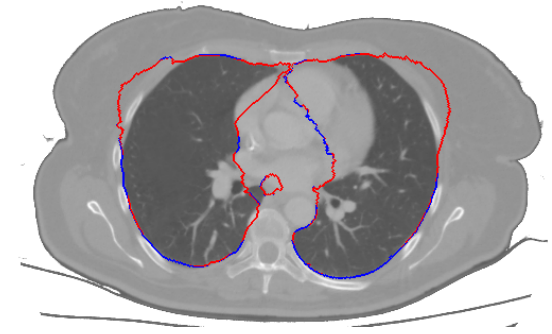

(e) prior term

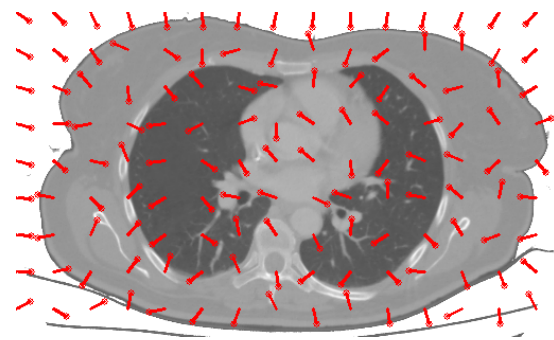

(c) vector field convolution

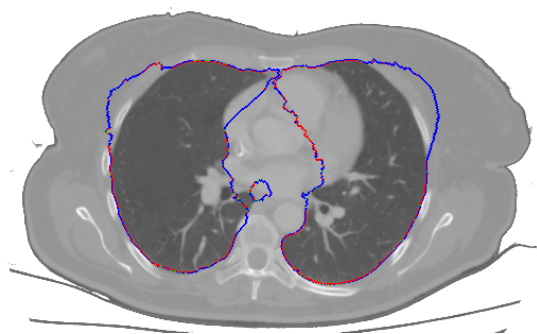

(f) edge term

Figure 2: A visualization of the different force terms. In the bottom row, the input image is overlapped with the current contour, which is colored according the force. Green means the force is close to zero, while blue and red colors mark inward and outward forces, respectively. In this example, the region term (d) is correctly moving the contour towards the lungs boundaries, as they define two very homogeneous areas. The prior term (e) is just pulling towards the prior segmentation (b). Finally, the edge term (f) is moving the level set towards the closest edges, regardless whether these belongs to the lungs boundaries or not.

the availability of a single image of a similar target object, along with its segmentation. The initial registration-based step provides a prior segmentation that will allow the LS to start its evolution near the area to be segmented. This benefits both the speed and the accuracy of the segmentation since, with a default initialization over the whole image, features located far from the target area are more likely to negatively influence the evolution of the LS.

The LS moves under the influence of three force terms, each providing information about a different characteristic of the current contour. There are a region, an edge and a prior term. The region term minimizes the inhomogeneity of the intensity values inside and outside the surface enclosed by the evolving contour, while the edge term attracts the curve towards natural boundaries and other edges of the image. Finally, the prior term moves the LS towards the prior segmentation obtained by the registration, incorporating the information gathered at the first stage of the method in the later segmentation process. Note that this is rather different than just using the prior as initial contour for the LS. Indeed, the prior term, rather than its initial location, influences the evolution of the contour, and can balance the other forces when they are small or inconsistent, leading to a more "conservative" segmentation with respect to using the initial contour.

Figure 3 provides an overview of HybridLS, while Figure 2 shows a visual example of the effect of three force terms in segmenting a lung CT. The total force acting on the LS is a linear combination of the force terms

$$
F_{\text {tot }}=w_{\mathrm{r}} F_{\mathrm{r}}(C)+w_{\mathrm{e}} F_{\mathrm{e}}(C)+w_{\mathrm{p}} F_{\mathrm{p}}(C, P)
$$

where $C$ is the current contour and $P$ is the prior segmentation. Along with the specific parameters for each term, the use of weights provides flexibility to our approach, allowing it to be adapted to the features and particularities of the objects to be segmented. In HybridLS, a GA is in charge of tuning the weights and the parameters of each term based on training data.

In what follows, we describe the components of HybridLS, starting with the computation of the registrationbased prior. Then, we define the three force terms and show how to compute them. Finally, we provide details about the GA and the parameter learning phase.

\subsection{Registration-based prior}

The registration algorithm we used is a recent contribution [41] called $\mathrm{SS}^{+}$. The optimization procedure, the core of the registration process, is based on the Scatter Search metaheuristic (section 2.2), which has been successfully used in a number of works in image registration [42]. In its original study on brain MRI, $\mathrm{SS}^{+}$provided superior results compared to other established techniques. Moreover, $\mathrm{SS}^{+}$delivered the best performance in a preliminary study on the registration of histological images.

In this work, the applications of $\mathrm{SS}^{+}$are extended to histological and CT images. The registration is performed in two steps, beginning with affine registration. Being a 
composition of translation, rotation, scaling and shearing operations, an affine transform can remove large misalignments between the images. Then, a deformable B-Splinebased registration takes care of adjusting the overlap locally, to match the finer details.

To compute a prior, one of the training images plays the role of the atlas. In cases where the target object has a large anatomical variability, a single atlas cannot express the whole distribution of possible shapes the object can assume, leading to poor registration results. This can be improved by using multiple atlases and selecting the most similar atlas for the registration to the target.

In HybridLS, the prior is obtained considering multiple images to be used as atlas. To select the actual atlas, all candidate images are registered to the target image using affine registration. Then, the candidate atlas having the highest similarity metric value is selected for the further B-Spline registration step.

\subsection{Force terms}

\subsubsection{Region term}

Our region term is borrowed from the classic "Active Contours Without Edges" (CV) [43] method by Chan and Vese. This algorithm was designed to detect objects whose boundaries are not necessarily defined by gray level gradients; indeed, it ignores edges completely, converting $\mathrm{CV}$ in a region-based method. The idea is to separate the image into two regions having homogeneous intensity values. More formally, the process minimizes the energy functional shown in Equation 2. The functional is used to evolve a LS representing the contour $C$, using the conventional variational calculus approach.

$$
\begin{aligned}
E(C)=\mu & \cdot \operatorname{Length}(C)+\nu \cdot \operatorname{Area}(C) \\
& +\lambda_{1} \int_{C}\left|I(x, y)-\bar{I}_{C}\right|^{2} d x d y+ \\
& +\lambda_{2} \int_{\Omega \backslash C}\left|I(x, y)-\bar{I}_{\Omega \backslash C}\right|^{2} d x d y
\end{aligned}
$$

In the equation, $I$ is the intensity value of the image to be segmented and $\bar{I}$ is its average value. Along with the length of $C$ and its area, there are a third and fourth term representing the variance of the intensity level (i.e., the homogeneity) inside and outside $C$. Each term has a weight that determines its influence on the total energy, so that, for instance, the smaller $\mu$, the more the length of the curve can increase without penalizing the minimization.

In HybridLS, we are interested in a pure region-based term without area or length restrictions, therefore we just use the two homogeneity terms. Therefore, in terms of force acting on the LS, we get

$$
F_{r}(x, y, C)= \begin{cases}\lambda_{1}\left|I(x, y)-\bar{I}_{C}\right|^{2} & (x, y) \in C \\ \lambda_{2}\left|I(x, y)-\bar{I}_{\Omega \backslash C}\right|^{2} & (x, y) \notin C\end{cases}
$$

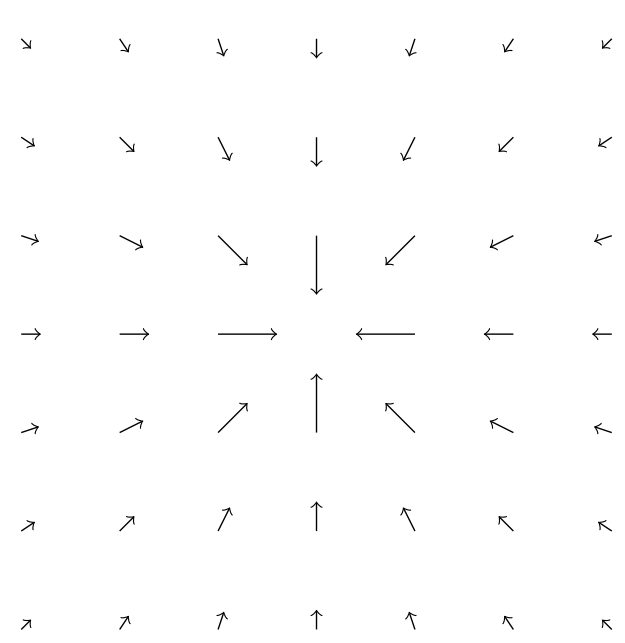

Figure 4: Vector field kernel used to compute the VFC term.

\subsubsection{Edge term}

The edge term incorporates the information about the boundaries in the image. Basically, the edge term pulls each point of the contour towards the closest edge. Our edge term is based on Vector Field Convolution (VFC) [44]. Compared to other edge-based forces such as Gradient Vector Flow [45], VFC has a lower computational cost and shows better robustness to noise and initialization. In addition, it showed good performance as external force for DMs [37, 44].

The VFC is static, in the sense that it does not depend on the current LS but only on the target image, therefore the field is calculated only once. The computation of the force occurs in two independent steps. First, an edge map of the target image is obtained applying Gaussian smoothing followed by the Sobel edge detector [46]. Then, the edge map is convolved with a vector field kernel $K$ in which each vector points to the origin, as in Figure 4 . The magnitude of the vectors decreases with the distance $d$, in such a way that distant edges produce a lower force than close edges (the actual value is $1 / d^{\gamma+1}$ ). For a point $c$ of contour $C$, the edge term is simply the normal component of the $\mathrm{VFC}$ with respect to $C$.

\subsubsection{Prior term}

The aim of the prior term is to move the LS towards the prior segmentation. Also, we want the module of every force vector to be proportional to the overlap between the current evolving curve and the prior segmentation. The idea about how to compute the actual force comes from the region term. If one considers the prior segmentation as a binary image, having an intensity value inside the object and another one outside, this image has two regions that are perfectly homogeneous. This is exactly the kind of result our region-based term was designed to deliver. Therefore, to compute the prior term we simply calculate the region term on the prior image, rather than on the target image. 


$$
F_{p}(x, y, C)= \begin{cases}\left|P(x, y)-\bar{P}_{C}\right|^{2} & (x, y) \in C \\ \left|P(x, y)-\bar{P}_{\Omega \backslash C}\right|^{2} & (x, y) \notin C\end{cases}
$$

In this case, $\lambda_{1}$ and $\lambda_{2}$ have been set to 1 , since the images used to calculate the prior term force are binary, thus presenting a perfectly homogeneous foreground and background, and it is not necessary to weight one region more heavily.

\subsubsection{Implementation}

In HybridLS, the contour $C$ evolves according to

$$
\frac{d C}{d t}=F_{\text {tot }} \cdot \vec{N}
$$

were $F_{\text {tot }}$ is the weighted sum of the three force terms (Equation 1) and $\vec{N}$ is the normal direction of $C$. We used the Shi and Karl's Fast-Two-Cycle (FTC) algorithm [47], a fast LS implementation without the need of solving partial differential equations (PDE). It is a narrow band technique that restricts the calculations of the LS to a much smaller region than the whole grid, and significantly speed up the curve evolution process. This method also separates the evolution process into two different cycles: one cycle for the data dependent term and a second cycle for the smoothness regularization.

\subsection{Parameter learning using metaheuristics}

HybridLS has the ability to learn optimal parameter settings for every specific dataset. Provided a training set of already segmented images of the same class, the parameters are learned using a classic machine learning approach: configurations of parameters are tested on the training data, and the results are compared with the ground truth to assess their quality. In the most basic approach, all combinations of parameters need to be tested, but this exhaustive search is very time consuming, if not even impossible when a large number of parameters are involved. Fortunately, we can overcome this problem by using metaheuristics, since a properly designed metaheuristic has the ability of learning optimal parameter values faster than an exhaustive search.

In this work, we developed a GA to learn the weights of the force terms $\left(w_{r}, w_{e}, w_{p}\right)$ and their corresponding parameters $\left(\lambda_{1}, \lambda_{2}\right.$ for the region term and $\gamma$ for the edge term). A solution of the problem, or an individual in GA terms, is a string of real values encoding the parameters values. The quality of a solution $s$ (its fitness) is defined as the average quality of the segmentations obtained using the parameters values in $s$. In this case, we measured the average Dice coefficient obtained segmenting the images in the training set.

The GA begins by creating a set of random solutions (a population) of fixed size. Then, individuals are selected and variation operators are applied to create a new generation of solutions. The current population is then entirely replaced by new one, except for the best individual that is never discarded (elitism). The individuals are selected using a tournament: $k$ individuals are drawn at random, and the best individual of the group (the winner of the tournament) is selected. The variation operators, responsible for combining and altering solutions, are blend crossover (BLX- $\alpha$ ) [48] and random mutation [49]. The random mutation operator picks randomly one of the individual parameters and replace it with a random value in the parameter's range, both times using uniform probability. Blend crossover operator is more complex: given two individuals $x$ and $y$, called "parents", for each position $i$ of the parents' coding, the algorithm computes the value $d=\left|x_{i}-y_{i}\right|$ and then randomly generates two values $a, b$ in the interval $\left[\min \left(x_{i}, y_{i}\right)-\alpha d, \max \left(x_{i}, y_{i}\right)+\alpha d\right]$ with uniform probability. The values $a$ and $b$ are assigned to the $i$-th positions of the two offspring, and $\alpha$ is a positive value controlling the width of the ranges in which the new parameters' values are drawn.

It is important to notice that, when testing combinations of parameter values, not all segmentation steps need all parameters. For instance, the VFC of an image depends only on $\gamma$. Having this in mind, and in order to speed up the learning process, we saved in a cache all the information that are shared between different configurations. This is especially important for the prior, which is the most computationally demanding step in the segmentation process by far. The prior do not use any of the parameters in the learning process, therefore only one per image is needed but, since the registration algorithm is non-deterministic, we represented its variability by creating a pool of 30 priors for each image. The priors used in tuning the segmentation parameters were drawn at random from the pool. This approach led to an impressive speedup of the training process. Once the priors and the VFC of each training image has been computed, a single parameter configuration could be tested in less than a second.

\section{Experimental Setup}

One of the main aims of this research is to develop a method that, combining the advantages of geometric DMs, metaheuristics and prior shape knowledge, can achieve remarkable results with different medical image modalities and anatomical structures of interest. To accomplish this purpose, three image modalities with completely different characteristics and various structures have been tested. In this section, these datasets will be described, as well as the anatomical structures to be segmented. Then, we will present the different methods included in the comparison, and devote two separate sections to the atlas registration and the tuning of the parameters (given their critical importance in our pipeline). Finally the final results of segmentation will be presented and analyzed. 


\subsection{Datasets}

Three kinds of biomedical image modalities were used to verify the global performance of the different methods over different datasets. We focused our interest on microscopy histological images derived using In Situ Hybridization, X-Ray computed tomography, and magnetic resonance imaging.

- In Situ Hybridization-derived images (ISH). 26 microscopy histological images were downloaded from the Allen Brain Atlas (ABA) [50]. The anatomical structure to segment was the hippocampus, and the ground truth was created manually by an expert in molecular biology: every image was manually segmented 5 times and, for each group of 5 manual segmentations, the consensus image was calculated and used as ground truth. The typical resolution of ABA images is about $15,000 \times 7,000$ pixels, and the ROIs about $2,500 \times 2,000$ pixels.

- Magnetic Resonance Imaging (MRI). A set of 17 $T_{1}$-weighted brain MRI were retrieved from a NMR database with their associated manual segmentations [51]. The deep brain structures to segment were caudate, putamen, globus pallidus, and thalamus. All MR images used in training and test have a resolution of $256 \times 256$ pixels.

- X-Ray Computed Tomography (CT). A set of 10 CT images were used in the experiments [37]. Four of them correspond to a human knee and the other six to human lungs. The gray value of all pixels have been inverted so the bone and the lungs are the darker objects in the image. Knee images have an average size of $410 \times 435$ pixels, while Lung images have $510 \times 350$ pixels.

All four datasets, considering lungs and knee as different image sets, were divided in training and test data. The training images were used by HybridLS for the learning of the parameters, while the test images were the ones used in the final experiments to check the performance of the methods.

In ISH, 22 images were used for testing and 4 as a training set. As atlas for the registration, the actual references in the ABA were employed to obtain the shape prior. With respect to MRI, 3 images were used as training set, another 3 were used as atlas, and the remaining 11 as test set. Finally, in relation to $\mathrm{CT}$, one image of every organ was used as training and atlas for the registration, leaving 3 lung and 2 knee images for testing the system.

\subsection{Methods included in the comparison}

In our comparisons we have included both deterministic and non-deterministic methods, as well as classic and very recent proposals. The stress has been focused on DMs, and their hybridization with metaheuristics, but other kinds of approaches have also been taken into account.
- Active Shape Models (and Iterative Otsu Thresholding Method) refined using Random Forests (ASM + RF) $[29,52]$. This method, published in 2012, uses a medial-based shape representation in polar coordinates, with the objective of creating simple models that can be managed in an easy and fast manner. Such a parametric model is moved and deformed by a metaheuristic (Differential Evolution (DE) [53]) according to an intensity-based similarity function between the model and the object itself. After that, Otsu's thresholding method [54] is iteratively applied on every region identified by the located control points. Finally, Random Forests [55] is applied to expand the segmented area to the regions that were not properly localized. This segmentation algorithm has shown very good performance in histological images, but needs a training set of shapes to manually create the parametric template and its possible deformations, as well as a training set of textural patterns for the expansion phase. Due to these restrictions it was only applied to ISH images.

- Soft Thresholding (ST) [56]. This deterministic method, presented in 2010, is based on relating each pixel in the image to the different regions via a membership function, rather than through hard decisions, and such a membership function is derived from the image histogram. In a first stage, the normalized histogram of the image is calculated and a sum of weighted known distributions is fit to it. Each probability distribution represents the probability for a pixel with a certain value to belong to the corresponding region. This segmentation technique is totally automatic, and the spatial operations performed make the thresholding more robust to noise and artifacts. Having been successfully applied to CT, MRI and ultrasound, it seemed interesting to apply it also to microscopy histological images and compare its performance with other state-of-the-art methods.

- Atlas-based deformable segmentation (DS) [41]. This method refers to the atlas-based segmentation procedure used in HybridLS to compute the prior (section 3.1). This is actually a stand-alone segmentation method, therefore it is included in the experimental study as a representative of registrationbased segmentation algorithms. Moreover, comparing DS's and HybridLS's results will assess the influence of the prior term on the performance of the second method. During the whole study, the setup and the atlas selection mechanism of DS (section 3.1) are always the same whether the method is used stand-alone or embedded in another segmentation technique.

- Geodesic Active Contours (GAC) [57]. This technique, introduced in 1997, connects classical 'snakes' based on energy minimization and geometric active 
contours based on the theory of curve evolution. It is based on active contours that evolve in time according to intrinsic geometric measures of the image: the evolving contours naturally split and merge, allowing the simultaneous detection of several objects and both interior and exterior boundaries.

The Partial Differential Equation of the GAC is the following:

$$
u_{t}=\alpha \cdot \operatorname{div}(g \nabla u /|\nabla u|)|\nabla u|+\beta \cdot g|\nabla u|
$$

where $g$ is a positive and strictly decreasing function, $\nabla$ is the gradient operator computed on image $I$, div is the divergence operator (that measures the magnitude of a vector field's source or sink at a given point), and $\alpha$ and $\beta$ are the contour (internal force) and expansion (external force) weights, respectively. This method is equivalent to the minimization of the length of curve $C$ according to a Riemannian metric, and such a metric depends here on the local gradient of the image $I$.

In this paper, two implementations of GAC have been tested. The first one uses as initial contour the whole image, while the second one, called DSGAC, employs the segmentation obtained using DS to create the initial contour of the geometric DM.

- Chan\&Vese Level Set Model (CV) [43]. This implicit DM was also included in the comparison to check its performance in comparison with the other approaches (see section 3.2.1). Also in this case, like in GAC, two implementations have been tested. The first one uses the whole image as initial contour, and the second one employs the segmentation result obtained by DS as the LS initial contour.

\subsection{Parameter settings}

As HybridLS has an automatic parameter learning phase, it would be unfair to compare it against other methods without some kind of parameter tuning. A manual tuning is time consuming and error-prone, while using the GA to tune all methods could introduce a bias, as the behavior of the GA could vary with each method. In general, we want the competitors to deliver their best performance, regardless of their parameter sensitivity or their ability to be tuned. Therefore, we decided to tune the competitors with an exhaustive search using the test data, rather then the training one. This means the results reported for all methods but HybridLS are actually the best average results they can obtain on these datasets. This gives them a clear advantage over HybridLS, as for the latter the parameters are learned using the training data only.

For CV, GAC, DSGAC and DSCV, all the possible combinations of the values in Table 1 were tested. Also, a pre-processing and a post-processing stages were included to improve the results obtained. The post-processing stage makes a refinement of the results removing the connected components smaller than a certain amount of pixels, while the pre-processing is a median filter to remove salt-andpepper-like noise present in some of the images. Moreover, for DSGAC and DSCV, 10 different initial masks were created using DS and the best one was used in the tuning. The number of iterations for GAC and $\mathrm{CV}$ was set to 500 to ensure the process reached convergence. In a few cases, on the ISH dataset, CV failed to converge within the limit due to poor parameters values. This occurred only while producing very low quality, degenerate segmentations, therefore the early stopping did not affect the tuning process.

After tuning these methods, the minimum allowed size in pixels of connected components was set to 75, 200 and 25000 for MRI, CT and ISH, respectively. For ASM+RF, the parameters used (Table 3 ) were those suggested in the bibliography for the ISH dataset, which has been deeply tested by the authors.

For HybridLS, the parameters settings were learned by the GA using the training data. The size of the population was set to 50 individuals, and the evolution lasted 50 generations. The probability of crossover and mutation was set to 0.7 and 0.1 , respectively, and the size of the tournament was 3 . The range of $\lambda_{1}, \lambda_{2}$ was restricted to $\{1,2,5\}$ to match the settings used with the other methods.

The final parameters configurations are reported in Table 2. It is interesting to remark how the GA detected a different level of importance for each term across the datasets. For instance, in MRI the edge term is not used $\left(w_{e}=0\right)$ since our machine learning system determines that, for a good segmentation, the region term and prior shape knowledge are enough. When segmenting CT lungs the only term used is the region-based one. In this case, $\lambda_{1}$ and $\lambda_{2}$ were set to 5 and 2 , respectively. This means that our final segmentation will have a more uniform foreground region (since the energy contributed by the "variance" in the foreground region has a larger weight), at the expense of allowing more variation in the background.

Table 1: Combination of parameters tested for CV, GAC, DSCV and DSGAC.

\begin{tabular}{cc} 
Parameter & Values \\
\hline$\alpha$ contour_weight & $\{1,2,3\}$ \\
$\beta$ expansion_weight & $\{-1,-0.5\}$ \\
$\mu$ weightLengthTerm & $\{0.01,0.1,0.25,0.5,0.75\}$ \\
$\lambda_{1}$ & $\{1,2,5\}$ \\
$\lambda_{2}$ & $\{1,3,5,10\}$ \\
size median filter & $\{1,50,75,100,200,5000,25000\}$
\end{tabular}

\subsection{Experimental results}

To evaluate the performance of the segmentation methods, we employed three standard segmentation metrics: the Dice coefficient (DSC), the Jaccard similarity index (JI) and the Hausdorff distance (HD). Both the Dice coefficient and the Jaccard index measure set agreement: a 
Table 2: Parameters obtained after tuning ST, GAC, CV, DS+GAC, DS+CV, and training HybridLS.

\begin{tabular}{lllll} 
CV & GAC & CV+DS & GAC + DS & HybridLS \\
\hline \multicolumn{4}{c}{ Magnetic Resonance Imaging } \\
\hline 500 iterations & 500 iterations & 500 iterations & 500 iterations & $\lambda_{1}=5$ \\
$\nu=0$ & $\beta=-1$ & $\nu=0$ & $\beta=-0.5$ & $\lambda_{2}=1$ \\
$\mu=0.01$ & $\alpha=3$ & $\mu=0.01$ & $\alpha=3$ & $w_{r}=5.1$ \\
$\lambda_{1}=\lambda_{2}=1$ & medFiltSize $=3$ & $\lambda_{1}=1$ & medFiltSize $=1$ & $w_{p}=1.1$ \\
medFiltSize $=1$ & & $\lambda_{2}=1$ & & $w_{e}=0$ \\
& & medFiltSize $=5$ & & $\gamma=1.5$
\end{tabular}

\begin{tabular}{|c|c|c|c|c|}
\hline \multicolumn{5}{|c|}{ Computerized Tomography - Knee } \\
\hline $\begin{array}{l}500 \text { iterations } \\
\nu=0 \\
\mu=0.01 \\
\lambda_{1}=5 \\
\lambda_{2}=2 \\
\text { medFiltSize }=3\end{array}$ & $\begin{array}{l}500 \text { iterations } \\
\alpha=1 \\
\beta=-0.5 \\
\text { medFiltSize }=1\end{array}$ & $\begin{array}{l}500 \text { iterations } \\
\nu=0 \\
\mu=0.01 \\
\lambda_{1}=1 \\
\lambda_{2}=1 \\
\text { medFiltSize }=1\end{array}$ & $\begin{array}{l}500 \text { iterations } \\
\alpha=3 \\
\beta=-0.5 \\
\text { medFiltSize }=1\end{array}$ & $\begin{array}{l}\lambda_{1}=2 \\
\lambda_{2}=5 \\
w_{r}=4.8 \\
w_{p}=0.9 \\
w_{e}=2 \\
\gamma=1.5\end{array}$ \\
\hline \multicolumn{5}{|c|}{ Computerized Tomography - Lungs } \\
\hline $\begin{array}{l}500 \text { iterations } \\
\nu=0 \\
\mu=0.01 \\
\lambda_{1}=5 \\
\lambda_{2}=2 \\
\text { medFiltSize }=3\end{array}$ & $\begin{array}{l}500 \text { iterations } \\
\beta=-1 \\
\alpha=2 \\
\text { medFiltSize }=3\end{array}$ & $\begin{array}{l}500 \text { iterations } \\
\nu=0 \\
\mu=0.01 \\
\lambda_{1}=1 \\
\lambda_{2}=5 \\
\text { medFiltSize }=3\end{array}$ & $\begin{array}{l}500 \text { iterations } \\
\beta=-1 \\
\alpha=3 \\
\text { medFiltSize }=3\end{array}$ & $\begin{array}{l}\lambda_{1}=5 \\
\lambda_{2}=2 \\
w_{r}=1.5 \\
w_{p}=0 \\
w_{e}=0 \\
\gamma=1.5\end{array}$ \\
\hline
\end{tabular}

\begin{tabular}{|c|c|c|c|c|}
\hline \multicolumn{5}{|c|}{ In Situ Hybridization-derived images } \\
\hline $\begin{array}{l}500 \text { iterations } \\
\nu=0 \\
\mu=0.01 \\
\lambda_{1}=\lambda_{2}=1 \\
\text { medFiltSize }=5\end{array}$ & $\begin{array}{l}500 \text { iterations } \\
\beta=-1 \\
\alpha=3 \\
\text { medFiltSize }=10\end{array}$ & $\begin{array}{l}500 \text { iterations } \\
\nu=0 \\
\mu=0.01 \\
\lambda_{1}=1 \\
\lambda_{2}=1 \\
\text { medFiltSize }=5\end{array}$ & $\begin{array}{l}500 \text { iterations } \\
\beta=-1 \\
\alpha=3 \\
\text { medFiltSize }=10\end{array}$ & $\begin{array}{l}\lambda_{1}=1 \\
\lambda_{2}=1 \\
w_{r}=1.9 \\
w_{p}=2.2 \\
w_{e}=1 \\
\gamma=2\end{array}$ \\
\hline
\end{tabular}

Table 3: Parameters used in ST, DS and ASM+RF. All parameters were taken from the original proposals.

\begin{tabular}{lll}
$\mathrm{ST}$ & $\mathrm{ASM}+\mathrm{RF}$ & $\mathrm{DS}$ \\
\hline L=2 regions & $C r=0.9$ & Metric = AdvancedNormalizedCorrelation \\
Relative max & $F=0.7$ & Optimizer = ScatterSearch \\
normalization & Uniform Crossover & $S S_{b}=12$ \\
& DE/target-to-best $/ 1$ & PSize $=32$ \\
& Population Size $=80$ & BLX- $\alpha=0.3$ \\
& Iterations $=250$ & LS-iterations $=25$ \\
& Median Filter $[25 \times 25]$ & NumberOfIterations $=15$ \\
& RF with 500 trees & NumberOfResolutions $=3$ \\
& & NumberOfSpatialSamples $=2000500010000$ \\
& Restarts $=3$
\end{tabular}


value of 0 indicates no overlap with the ground truth, and a value of 1 indicates perfect agreement. In turn, the Hausdorff distance measures the mutual proximity of two images, calculated as the maximum distance from a point in the ground truth to the closest point in the segmentation.

It is important to remark that $\mathrm{ASM}+\mathrm{RF}, \mathrm{DS}, \mathrm{DSCV}$, DSGAC and HybridLS are non-deterministic, since stochastic methods, like Differential Evolution or Scatter Search, are embedded in these algorithms. It is essential to execute such algorithms several times to estimate and compare their performances. In this case, 20 repetitions per image were run and the mean, median and standard deviation values were calculated over the whole set of results (see Table 5). For instance, in ISH the mean Dice value of DS is 0.876 , and represents the average of 440 experiments performed (20 repetitions per image and 22 images).

We also performed a statistical analysis of the results. When comparing two methods, we used Wilcoxon ranksum test [58], a non-parametric statistical test that checks whether one of two independent samples tends to have larger values than the other. When multiple comparisons were performed, Holm correction [59] was applied to the $p$ values to control the family-wise error rate. Note that, in the Lungs and Knee datasets, the number of images is not large enough to allow the comparison of the deterministic methods (ST, CV and GAC), therefore they have been excluded from the test.

In Table 4, some concise information about the running time of each algorithm is provided with an illustrative purpose. The fastest method is ST with MRI, taking only 1 second per image, while the slowest are the different applications of DS to ISH, employing up to 10 minutes to process an image. Nevertheless, several factors affect the accuracy of a comparison in terms of execution time. First, some of the methods have been developed in MATLAB and others in $\mathrm{C}++$. Moreover, the size of the images differs from one image modality to another, as well as some of the pre- and post-processing stages we used. Finally, the nature of the algorithms is completely different, making them hardly comparable.

\subsubsection{Analysis}

The experimental results are reported in Table 5. Visual examples of two segmentations obtained by the methods on each dataset are provided in Figure 6. For simplicity, our discussion focuses on the results in terms of mean DSC, but note that this choice does not really affect the outcome of the comparison, as there is an almost perfect agreement with the other validation measures we considered.

The performance of the segmentation methods varies greatly across the four datasets. The easiest problem to be solved has been the segmentation of Lungs in CT images, with all methods but GAC and DS scoring higher than 0.95. The most complex task has shown to be the segmentation of deep anatomical structures in brain MRI, where four of the compared methods have obtained an average DSC of 0.2 or less).

The per-dataset results are shown in Figure 5 using boxplots and in Table 6 through the average rankings. Obviously, the performance of every method depends on the nature of the image to be segmented. For instance, techniques based on grey intensity level (such as CV and ST) yielded worse results in image modalities with less contrast and small differences in terms of pixel intensity like MRI.

HybridLS has obtained the best results in all biomedical image datasets. It achieved the best values for the mean DSC and JI metrics, and it was ranked as the best method in every image modality. The Wilcoxon test (Table 6) showed, with really high confidence, that the difference between HybridLS and the other methods is statistically significant in all but one case (DS on MRI). This behavior is also robust, as shown by the low standard deviation values. We can then conclude that our proposal is the best segmentation method in the comparison.

The DS method has been one of the best-performing algorithms, ranking second or third over three datasets. More in general, all methods using the registration-based initialization scored better than those using a standard one. This applies also to $\mathrm{CV}$ and GAC: in all but one case, both DSCV and DSGAC ranked better than their counterpart, with a statistically significant difference (Table 7$)$.

Overall, DSGAC delivered an acceptable performance, ranking above average in three datasets out of four. This is remarkable, as the regular GAC ranked constantly in the last three positions, and it can be explained by the high sensitivity of GAC to its initialization.

DSCV ranked around average in all datasets, performing slightly worse, although more consistently, than DSGAC. The plain CV method achieved a bad performance, ranking last or second to last in three datasets. Only on the Lungs dataset, where the grey value is enough to segment the target quite accurately, CV delivered good results.

$\mathrm{ST}$ results showed a similar pattern to $\mathrm{CV}$. It performed better than CV, but being ST based on the histogram it showed limited ability to cope with complex scenarios. On the other hand, ST is the fastest method on the group and it has virtually no parameters to be set.

$\mathrm{ASM}+\mathrm{RF}$ obtained some of the best results with ISH images, being also one of the fastest techniques, but it is fair to underline its ad-hoc nature. It needs a training set of shapes to create the template and its possible deformations, and it also needs a training set of textural patterns for the expansion phase. Also, it is not able to manage topological changes in a natural way, as geometric DMs can do.

Finally, from the values of $\mathrm{HD}$, it is interesting to notice how methods without shape deformation restrictions, like ST, CV and GAC, have a higher (worse) HD with respect to others introducing prior shape knowledge, like ASM+RF, DS and HybridLS. 
Table 4: Average execution time per method and kind of image. All values are in seconds, and were obtained running the experiments in an Intel Core i5-2410M @ 2.3GHz with $4.00 \mathrm{~GB}$ of RAM. Also the programming environment has been included between brackets.

\begin{tabular}{|c|c|c|c|c|c|c|c|c|}
\hline & $\begin{array}{c}\text { ASM+RF } \\
(\text { MATLAB }, \mathrm{C}++)\end{array}$ & $\begin{array}{c}\text { ST } \\
\text { (MATLAB) }\end{array}$ & $\begin{array}{c}\text { CV } \\
\text { (MATLAB) } \\
\end{array}$ & $\begin{array}{c}\text { GAC } \\
(\text { MATLAB }, \mathrm{C}++)\end{array}$ & $\begin{array}{c}\text { DSCV } \\
(\text { MATLAB }, \mathrm{C}++) \\
\end{array}$ & $\begin{array}{c}\text { DSGAC } \\
(\mathrm{MATLAB}, \mathrm{C}++) \\
\end{array}$ & $\begin{array}{c}\mathrm{DS} \\
(\mathrm{C}++) \\
\end{array}$ & $\begin{array}{c}\text { HybridLS } \\
(\mathrm{C}++) \\
\end{array}$ \\
\hline ISH & 35 & 39 & 87 & 32 & 582 & 493 & 471 & 545 \\
\hline Lungs & - & 1.7 & 36 & 15 & 384 & 342 & 326 & 331 \\
\hline Knee & - & 2.5 & 67 & 16 & 310 & 265 & 245 & 252 \\
\hline MRI & - & 1 & 11 & 1.5 & 429 & 407 & 404 & 405 \\
\hline
\end{tabular}

Table 5: Segmentation Results using 3 different metrics: Dice Similarity Coefficient (DSC), Jaccard Index (JI), and Hausdorff Distance (HD). Values are sorted in descending order using as criterion their average DSC value. The best results for every metric are shown in bold letters.

\begin{tabular}{|c|c|c|c|c|c|c|c|c|c|c|}
\hline \multirow{2}{*}{ Dataset } & \multirow{2}{*}{ Method } & \multicolumn{3}{|c|}{ Dice Coefficient } & \multicolumn{3}{|c|}{ Jaccard Index } & \multicolumn{3}{|c|}{ Hausdorff Distance } \\
\hline & & mean & median & stdev & mean & median & stdev & mean & median & stdev \\
\hline \multirow{8}{*}{ ISH } & HybridLS & 0.888 & 0.918 & 0.079 & 0.806 & 0.849 & 0.109 & 103.614 & 70.109 & 100.115 \\
\hline & $\mathrm{ASM}+\mathrm{RF}$ & 0.885 & 0.896 & 0.040 & 0.797 & 0.812 & 0.061 & 114.906 & 94.736 & 52.785 \\
\hline & DS & 0.876 & 0.907 & 0.078 & 0.787 & 0.829 & 0.108 & 101.437 & 75.166 & 79.779 \\
\hline & DSGAC & 0.791 & 0.830 & 0.143 & 0.674 & 0.709 & 0.172 & 215.237 & 150.727 & 196.908 \\
\hline & $\mathrm{ST}$ & 0.728 & 0.775 & 0.175 & 0.597 & 0.632 & 0.192 & 578.876 & 665.301 & 303.314 \\
\hline & DSCV & 0.673 & 0.764 & 0.203 & 0.538 & 0.618 & 0.203 & 263.345 & 176.706 & 234.192 \\
\hline & GAC & 0.670 & 0.722 & 0.181 & 0.528 & 0.564 & 0.192 & 693.257 & 707.560 & 265.589 \\
\hline & $\mathrm{CV}$ & 0.589 & 0.723 & 0.257 & 0.460 & 0.567 & 0.242 & 839.800 & 844.773 & 331.804 \\
\hline \multirow{7}{*}{ Knee } & HybridLS & 0.868 & 0.872 & 0.087 & 0.777 & 0.782 & 0.136 & 13.690 & 13.618 & 1.735 \\
\hline & DSGAC & 0.810 & 0.811 & 0.142 & 0.705 & 0.705 & 0.204 & 84.611 & 84.611 & 4.040 \\
\hline & DS & 0.687 & 0.685 & 0.227 & 0.569 & 0.563 & 0.271 & 45.015 & 45.308 & 25.303 \\
\hline & DSCV & 0.528 & 0.527 & 0.079 & 0.363 & 0.361 & 0.073 & 69.642 & 76.261 & 16.468 \\
\hline & GAC & 0.486 & 0.486 & 0.310 & 0.349 & 0.349 & 0.276 & 187.087 & 187.087 & 29.528 \\
\hline & $\mathrm{ST}$ & 0.398 & 0.398 & 0.088 & 0.250 & 0.250 & 0.069 & 144.864 & 144.864 & 36.792 \\
\hline & $\mathrm{CV}$ & 0.230 & 0.230 & 0.072 & 0.131 & 0.131 & 0.046 & 179.680 & 179.680 & 30.499 \\
\hline \multirow{7}{*}{ Lungs } & HybridLS & 0.996 & 0.997 & 0.001 & 0.992 & 0.994 & 0.003 & 2.949 & 3.606 & 1.415 \\
\hline & ST & 0.979 & 0.990 & 0.023 & 0.960 & 0.981 & 0.044 & 51.550 & 60.208 & 42.882 \\
\hline & $\mathrm{CV}$ & 0.973 & 0.992 & 0.034 & 0.949 & 0.983 & 0.063 & 52.903 & 60.208 & 38.592 \\
\hline & DSCV & 0.966 & 0.985 & 0.034 & 0.935 & 0.971 & 0.062 & 26.512 & 25.495 & 9.321 \\
\hline & DSGAC & 0.950 & 0.952 & 0.027 & 0.906 & 0.908 & 0.049 & 37.828 & 29.155 & 27.197 \\
\hline & DS & 0.896 & 0.897 & 0.062 & 0.818 & 0.814 & 0.102 & 39.378 & 51.478 & 18.023 \\
\hline & GAC & 0.670 & 0.627 & 0.251 & 0.542 & 0.457 & 0.309 & 143.938 & 137.568 & 34.430 \\
\hline \multirow{7}{*}{ MRI } & HybridLS & 0.758 & 0.780 & 0.048 & 0.612 & 0.639 & 0.062 & 7.819 & 6.708 & 2.874 \\
\hline & DS & 0.752 & 0.783 & 0.056 & 0.606 & 0.643 & 0.071 & 10.838 & 10.000 & 3.192 \\
\hline & DSGAC & 0.585 & 0.613 & 0.087 & 0.418 & 0.442 & 0.084 & 25.113 & 25.495 & 2.414 \\
\hline & DSCV & 0.204 & 0.213 & 0.054 & 0.115 & 0.119 & 0.033 & 76.792 & 76.322 & 5.955 \\
\hline & $\mathrm{CV}$ & 0.155 & 0.171 & 0.042 & 0.084 & 0.093 & 0.024 & 93.727 & 93.408 & 4.670 \\
\hline & $\mathrm{ST}$ & 0.175 & 0.181 & 0.053 & 0.097 & 0.100 & 0.032 & 84.936 & 85.586 & 6.289 \\
\hline & GAC & 0.124 & 0.139 & 0.035 & 0.066 & 0.074 & 0.020 & 90.856 & 90.139 & 5.459 \\
\hline
\end{tabular}




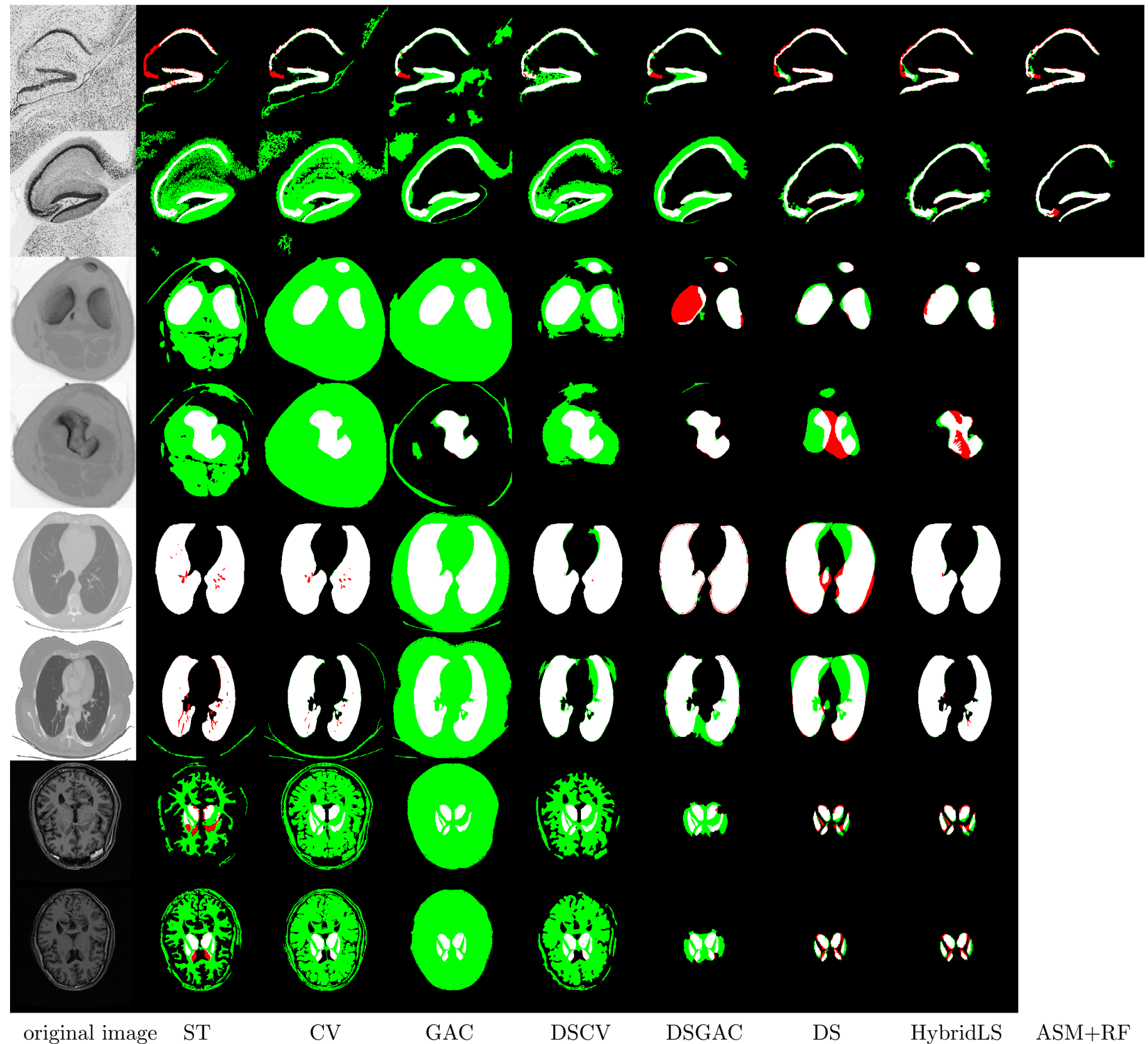

Figure 6: Some visual examples of the results obtained. Two images per image modality and structure to segment have been selected: the first two rows correspond to ISH, the next two rows to CT-Knee, and the last four to CT-Lungs and MRI. White represents true positives, red false negatives, and green false positives. 
Table 6: Average rank achieved by every method per image modality and adjusted p-value of Wilcoxon test comparing each algorithm against HybridLS.

\begin{tabular}{|c|c|c|c|}
\hline Dataset & Method & Mean Rank & $p$-value \\
\hline \multirow{8}{*}{ ISH } & HybridLS & 1.82 & \\
\hline & DS & 2.50 & 0.000 \\
\hline & $\mathrm{ASM}+\mathrm{RF}$ & 2.64 & 0.000 \\
\hline & DSGAC & 4.14 & 0.000 \\
\hline & $\mathrm{ST}$ & 5.68 & 0.000 \\
\hline & DSCV & 6.23 & 0.000 \\
\hline & GAC & 6.36 & 0.000 \\
\hline & $\mathrm{CV}$ & 6.64 & 0.000 \\
\hline \multirow{7}{*}{ Knee } & HybridLS & 1.50 & \\
\hline & DSGAC & 2.00 & 0.000 \\
\hline & DS & 3.00 & 0.000 \\
\hline & DSCV & 4.50 & 0.000 \\
\hline & GAC & 5.00 & - \\
\hline & $\mathrm{ST}$ & 5.50 & - \\
\hline & $\mathrm{CV}$ & 6.50 & - \\
\hline \multirow{7}{*}{ Lungs } & HybridLS & 1.00 & \\
\hline & $\mathrm{ST}$ & 2.33 & - \\
\hline & $\mathrm{CV}$ & 3.00 & - \\
\hline & DSCV & 3.67 & 0.000 \\
\hline & DSGAC & 5.33 & 0.000 \\
\hline & DS & 5.67 & 0.000 \\
\hline & GAC & 7.00 & - \\
\hline \multirow{7}{*}{ MRI } & HybridLS & 1.27 & \\
\hline & DS & 1.73 & 0.46 \\
\hline & DSGAC & 3.00 & 0.000 \\
\hline & DSCV & 4.18 & 0.000 \\
\hline & $\mathrm{ST}$ & 5.09 & 0.000 \\
\hline & $\mathrm{CV}$ & 5.73 & 0.000 \\
\hline & GAC & 7.00 & 0.000 \\
\hline
\end{tabular}

Table 7: Pairwise comparison of all the methods but HybridLS. Each cell of the table reports the p-value of Wilcoxon test comparing the method on the corresponding row with that on the column.

ISH

\begin{tabular}{rrrrrrr} 
ASH & ASMF & CV & DS & DSCV & DSGAC & GAC \\
\hline CV & 0.00 & & & & & \\
DS & 0.01 & 0.00 & & & & \\
DSCV & 0.00 & 0.38 & 0.00 & & & \\
DSGAC & 0.00 & 0.00 & 0.00 & 0.00 & & \\
GAC & 0.00 & 0.92 & 0.00 & 0.92 & 0.00 & \\
ST & 0.00 & 0.32 & 0.00 & 0.61 & 0.26 & 0.61
\end{tabular}

Knee

\begin{tabular}{rrr} 
& DS & DSCV \\
\hline DSCV & 0.00 & \\
DSGAC & 0.00 & 0.00
\end{tabular}

Lungs

\begin{tabular}{lrc}
\multicolumn{1}{l}{ Dungs } & DSCV \\
\hline DSCV & 0.00 & \\
DSGAC & 0.00 & 0.00
\end{tabular}

MRI

\begin{tabular}{rrrrrr}
\multicolumn{1}{l}{ MRI } & CV & DS & DSCV & DSGAC & GAC \\
\hline DS & 0.00 & & & & \\
DSCV & 0.02 & 0.00 & & & \\
DSGAC & 0.00 & 0.00 & 0.00 & & \\
GAC & 0.10 & 0.00 & 0.00 & 0.00 & \\
ST & 0.79 & 0.00 & 0.26 & 0.00 & 0.08
\end{tabular}

ISH
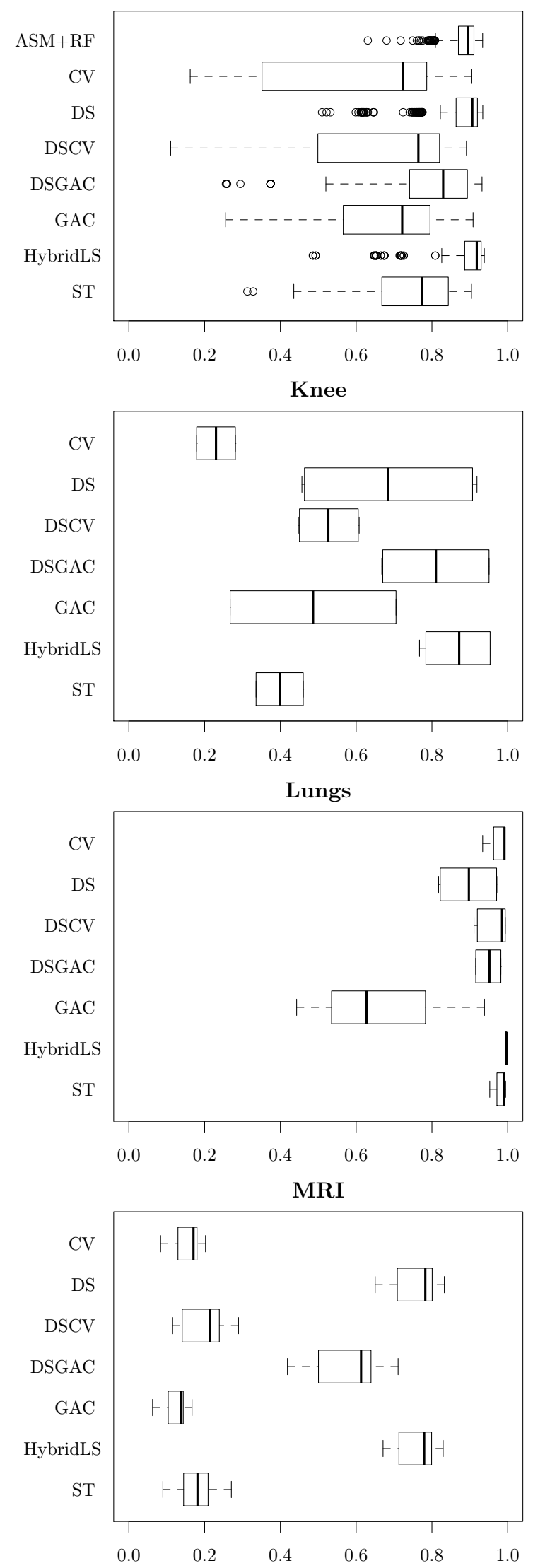

Figure 5: Box-plot representing the DSC for all methods. 


\section{Discussion and Future Research}

It is crucial to highlight the main features of HybridLS:

- it is an accurate and also general segmentation method (it obtains very good results with all the medical image modalities tested, even overcoming well-consolidated techniques);

- its overall standard deviation is the lowest among the different methods compared, therefore we can affirm that the developed approach is consistent and stable in terms of performance;

- it does not need a training set of textures or shapes to segment the object of interest (it needs only one reference image to obtain the shape prior);

- it performs self-adaptation of its own parameters depending on the medical image modality to segment;

- and uses metaheuristics to generate the shape prior and to perform the previously mentioned learning of parameters.

Thanks to the automatic learning of the model parameters, our hybrid proposal is able to perform an effective segmentation with very different medical image modalities, adapting the importance of every term to each image modality and anatomical structure.

The main drawback of HybridLS is that it is not as fast as $\mathrm{ST}$ or even $\mathrm{ASM}+\mathrm{RF}$. This is obvious since it can be as fast as its components and, evidently, DS is a deformable registration process that can take several minutes in a general purpose computer. More sophisticated implementations, like GPGPU programming, can be tested to speed-up the computations. Finally, the introduction of a textural term could be taken into consideration if the benefits obtained with its use justify it.

\section{Acknowledgments}

Pablo Mesejo and Andrea Valsecchi are funded by the European Commission (Marie Curie ITN MIBISOC, FP7 PEOPLE-ITN-2008, GA n. 238819). The authors want to thank Nicola Bova for his generous and helpful advice on the development of the level set implementation. NMR database is the property of "CEA/I2BM/NeuroSpin" and can be provided on demand to cyril.poupon@cea.fr. Data were acquired with PTK pulse sequences, reconstructed with PTK reconstructor package and postprocessed with Brainvisa/Connectomist software, freely available at http: //brainvisa.info.

\section{References}

[1] D. L. Pham, C. Xu, J. L. Prince, Current Methods in Medical Image Segmentation, Annual Review of Biomedical Engineering 2 (2000) 315-337.
[2] L. He, Z. Peng, B. Everding, X. Wang, C. Y. Han, K. L. Weiss, W. G. Wee, A comparative study of deformable contour methods on medical image segmentation, Image and Vision Computing 26 (2008) 141-163.

[3] T. Heimann, H.-P. Meinzer, Statistical shape models for 3D medical image segmentation: a review, Medical Image Analysis 13 (2009) 543-563.

[4] J. Malik, S. Belongie, T. Leung, J. Shi, Contour and Texture Analysis for Image Segmentation, International Journal of Computer Vision 43 (1) (2001) 7-27, ISSN 0920-5691.

[5] Y. Zhang, B. J. Matuszewski, L.-K. Shark, C. J. Moore, Medical Image Segmentation Using New Hybrid Level-Set Method, in: Procs. of the International Conference BioMedical Visualization: Information Visualization in Medical and Biomedical Informatics, 71-76, 2008.

[6] M. Chupin, A. Hammers, R. S. N. Liu, O. Colliot, J. Burdett, E. Bardinet, J. S. Duncan, L. Garnero, L. Lemieux, Automatic segmentation of the hippocampus and the amygdala driven by hybrid constraints: Method and validation, NeuroImage 46 (3) (2009) 749-761

[7] M. Gendreau, J.-Y. Potvin, Handbook of Metaheuristics, Springer Publishing Company, Incorporated, 2nd edn., ISBN 1441916636, 9781441916631, 2010.

[8] D. E. Goldberg, Genetic Algorithms in Search, Optimization and Machine Learning, Addison-Wesley, 1989.

[9] M. Laguna, R. Martí, Scatter search: methodology and implementations in C, Kluwer Academic Publishers, Boston, 2003.

[10] D. Terzopoulos, K. Fleischer, Deformable Models, The Visual Computer 4 (1988) 306-331.

[11] M. Kass, A. Witkin, D. Terzopoulos, Snakes: Active contour models, International Journal of Computer Vision 1 (1988) 321331.

[12] T. F. Cootes, C. J. Taylor, D. H. Cooper, J. Graham, Active shape models-their training and application, Computer Vision and Image Understanding 61 (1995) 38-59.

[13] T. F. Cootes, G. J. Edwards, C. J. Taylor, Active Appearance Models, in: Proc. of the European Conference on Computer Vision, vol. 2, 484-498, 1998.

[14] T. F. Cootes, G. Edwards, C. Taylor, Comparing Active Shape Models with Active Appearance Models, in: Procs. of British Machine Vision Conference, 173-182, 1999.

[15] M. Bro-Nielsen, Active nets and cubes, Tech. Rep., 1994.

[16] B. B. Kimia, A. R. Tannenbaum, S. W. Zucker, Shapes, Shocks, and Deformations I: The Components of Two-Dimensional Shape and the Reaction-Diffusion Space, International Journal of Computer Vision 15 (1994) 189-224.

[17] R. Kimmel, A. Amir, A. M. Bruckstein, Finding Shortest Paths on Surfaces Using Level Sets Propagation, IEEE Trans. on Pattern Analysis and Machine Intelligence 17 (6) (1995) 635-640.

[18] G. Sapiro, A. Tannenbaum, Affine Invariant Scale-Space, International Journal of Computer Vision 11 (1) (1993) 25-44.

[19] S. Osher, J. Sethian, Fronts propagating with curvaturedependent speed: algorithms based on Hamilton-Jacobi formulations, Journal of Computational Physics 79 (1) (1988) 12-49.

[20] J. Sethian, Level Set Methods and Fast Marching Methods: Evolving Interfaces in Computational Geometry, Fluid Mechanics, Computer Vision, and Materials Science, Cambridge Monographs on Applied and Computational Mathematics, Cambridge University Press, 1999.

[21] S. J. Osher, R. P. Fedkiw, Level Set Methods and Dynamic Implicit Surfaces, Springer, 2002.

[22] S. Luke, Essentials of Metaheuristics, Lulu, available for free at http://cs.gmu.edu/ sean/book/metaheuristics/, 2009.

[23] B. Zitová, J. Flusser, Image registration methods: a survey, Image and Vision Computing 21 (2003) 977-1000.

[24] J. P. W. Pluim, J. B. A. Maintz, M. A. Viergever, Mutualinformation-based registration of medical images: a survey, IEEE T. Med. Imaging 22 (8) (2003) 986-1004.

[25] F. Maes, D. Vandermeulen, P. Suetens, Comparative evaluation of multiresolution optimization strategies for image registration by maximization of mutual information, Medical Image Analy- 
sis 3 (4) (1999) 373-386.

[26] S. Klein, M. Staring, J. P. W. Pluim, Evaluation of Optimization Methods for Nonrigid Medical Image Registration Using Mutual Information and B-Splines, IEEE Trans. on Image Processing 16 (12) (2007) 2879-2890.

[27] S. Damas, O. Cordón, J. Santamaría, Medical Image Registration Using Evolutionary Computation: An Experimental Survey, IEEE Computational Intelligence Magazine 6 (4) (2011) 26 -42 .

[28] M. Cabezas, A. Oliver, X. Lladó, J. Freixenet, M. B. Cuadra, A review of atlas-based segmentation for magnetic resonance brain images, Computer Methods and Programs in Biomedicine 104 (3) (2011) e158 - e177.

[29] P. Mesejo, R. Ugolotti, F. D. Cunto, M. Giacobini, S. Cagnoni, Automatic Hippocampus Localization in Histological Images using Differential Evolution-Based Deformable Models, Pattern Recognition Letters 34 (3) (2013) 299 - 307.

[30] M. Heydarian, M. Noseworthy, M. Kamath, C. Boylan, W. Poehlman, Optimizing the Level Set Algorithm for Detecting Object Edges in MR and CT Images, IEEE Trans. on Nuclear Science 56 (1) (2009) $156-166$.

[31] L. Ballerini, Genetic Snakes for Medical Images Segmentation, in: Evolutionary Image Analysis, Signal Processing and Telecommunications, vol. 1596, 59-73, 1999.

[32] D.-H. Chen, Y.-N. Sun, A self-learning segmentation framework - the Taguchi approach, Computerized Medical Imaging and Graphics 24 (5) (2000) $283-296$.

[33] C. McIntosh, G. Hamarneh, Medial-based Deformable Models in Non-convex Shape-spaces for Medical Image Segmentation using Genetic Algorithms, IEEE Trans. on Medical Imaging 31 (1) (2012) 33-50.

[34] C.-Y. Hsu, C.-Y. Liu, C.-M. Chen, Automatic segmentation of liver PET images, Computerized Medical Imaging and Graphics 32 (7) (2008) $601-610$.

[35] O. Ibáñez, N. Barreira, J. Santos, M. G. Penedo, Genetic approaches for topological active nets optimization, Pattern Recognition 42 (5) (2009) 907-917.

[36] J. Novo, J. Santos, M. G. Penedo, Topological Active Models optimization with Differential Evolution, Expert Systems with Applications 39 (15) (2012) 12165-12176.

[37] N. Bova, Ó. Ibáñez, O. Cordón, Image Segmentation Using Extended Topological Active Nets Optimized by Scatter Search, IEEE Computational Intelligence Magazine 8 (1) (2013) 16-32.

[38] P. Ghosh, M. Mitchell, J. A. Tanyi, A. Hung, A Genetic Algorithm-Based Level Set Curve Evolution for Prostate Segmentation on Pelvic CT and MRI Images, in: Biomedical Image Analysis and Machine Learning Technologies, 127-149, 2010.

[39] D. Feltell, L. Bai, 3D level set image segmentation refined by intelligent agent swarm, in: Procs. of IEEE Congress on Evolutionary Computation, 1-8, 2010.

[40] P. Mesejo, S. Cagnoni, An experimental study on the automatic segmentation of in situ hybridization-derived images, in: Proc. on 1st International Conference on Medical Imaging using BioInspired and Soft Computing (MIBISOC'13), in Press, 2013.

[41] A. Valsecchi, S. Damas, J. Santamaría, L. Marrakchi-Kacem, Intensity-based Image Registration using Scatter Search, Tech. Rep. AFE 2012-14, URL http://docs.softcomputing.es/ public/afe/TR-AFE-2012-14.pdf, submitted to Artificial Intelligence in Medicine, 2012.

[42] O. Cordón, S. Damas, J. Santamaría, R. Martí, Scatter Search for the Point-Matching Problem in 3D Image Registration, INFORMS Journal on Computing 20 (1) (2008) 55-68.

[43] T. F. Chan, L. A. Vese, Active Contours without Edges, IEEE Trans. on Image Processing 10 (2001) 266-277.

[44] B. Li, S. Member, S. T. Acton, S. Member, Active contour external force using vector field convolution for image segmentation, IEEE Trans. on Image Processing 16 (2007) 2096-2106.

[45] C. Xu, J. L. Prince, Gradient Vector Flow: A New External Force for Snakes, in: Procs. of IEEE Conference on Computer Vision and Pattern Recognition, 66-71, 1997.

[46] R. C. Gonzalez, R. E. Woods, Digital Image Processing,
Addison-Wesley, 2nd edn., 2001.

[47] Y. Shi, W. C. Karl, A Real-Time Algorithm for the Approximation of Level-Set-Based Curve Evolution, IEEE Trans. on Image Processing 17 (5) (2008) 645-656.

[48] L. J. Eshelman, J. D. Schaffer, Real-coded genetic algorithms and interval-schemata., in: D. L. Whitley (Ed.), Foundation of Genetic Algorithms 2, Morgan Kaufmann., San Mateo, CA, 187-202, 1993.

[49] T. Bäck, D. B. Fogel, Z. Michalewicz, Handbook of Evolutionary Computation, IOP Publishing Ltd and Oxford University Press, 1997.

[50] Allen Institute for Brain Science, Allen Reference Atlases, http: //mouse.brain-map.org, 2004-2006.

[51] C. Poupon, F. Poupon, L. Allirol, J.-F. Mangin, A database dedicated to anatomo-functional study of human brain connectivity, in: Procs. of the Annual Meeting of the Organization for Human Brain Mapping, 646, 2006.

[52] P. Mesejo, R. Ugolotti, S. Cagnoni, F. Di Cunto, M. Giacobini, Automatic Segmentation of Hippocampus in Histological Images of Mouse Brains using Deformable Models and Random Forest, in: Procs. of Symposium on Computer-Based Medical Systems, 2012.

[53] S. Das, P. Suganthan, Differential Evolution: A Survey of the State-of-the-Art, IEEE Trans. on Evolutionary Computation 15 (2011) 4-31.

[54] N. Otsu, A Threshold Selection Method from Gray-Level Histograms, IEEE Trans. on Systems, Man and Cybernetics 9 (1) (1979) $62-66$.

[55] L. Breiman, Random Forests, Maching Learning 45 (2001) 5-32.

[56] S. Aja-Fernandez, G. Vegas-Sanchez-Ferrero, M. Martin Fernandez, Soft thresholding for medical image segmentation, in: Proc. International Conference of the IEEE Engineering in Medicine and Biology Society (EMBC), 4752 -4755, 2010.

[57] V. Caselles, R. Kimmel, G. Sapiro, Geodesic Active Contours, International Journal of Computer Vision 22 (1997) 61-79.

[58] W. H. Kruskal, Historical Notes on the Wilcoxon Unpaired TwoSample Test, Journal of the American Statistical Association 52 (279) (1957) 356-360.

[59] S. Holm, A Simple Sequentially Rejective Multiple Test Procedure, Scandinavian Journal of Statistics 6 (2) (1979) 65-70. 